\title{
Atomistic Modeling of Anisotropic Mechanical Properties of Lanthanum Zirconate Nanocystal
}

\author{
Xingye Guo ${ }^{\mathrm{a}, \mathrm{c}}$, Jian Zhang ${ }^{\mathrm{a}}$, Hye-Yeong Park ${ }^{\mathrm{b}}$, Yeon-Gil Jung ${ }^{\mathrm{b}}$, Jing Zhang ${ }^{\mathrm{a} *}$ \\ a Department of Mechanical and Energy Engineering, Indiana University-Purdue University \\ Indianapolis, IN 46202, USA \\ b School of Materials Science and Engineering, Changwon National University, Changwon, \\ Kyungnam 641-773, Republic of Korea \\ c College of Materials Science and Engineering, Beijing University of Technology, Beijing, \\ 100124, China \\ *corresponding authors: jz29@iupui.edu
}

\begin{abstract}
Lanthanum zirconate $\left(\mathrm{La}_{2} \mathrm{Zr}_{2} \mathrm{O}_{7}\right.$, or $\left.\mathrm{LZ}\right)$ has been widely recognized as a promising candidate material for thermal barrier coating (TBC) applications since it has low thermal conductivity, high-temperature phase stability, and low sintering activity. However, the mechanical properties of LZ crystal have not been fully understood, which hinders it from future applications. In this work, atomistic simulations were performed to study the anisotropic mechanical properties of LZ crystal. Using both the first principles and molecular dynamics (MD) calculations, uniaxial tensile tests of LZ crystal in [001], [011], and [111] directions were simulated. The stress-strain curves of the tensile tests were calculated, and the ultimate tensile strength and toughness were derived. The Young's moduli in [001], [011], and [111] directions were calculated using both the stress-strain curves and an analytical method for small deformation. Additionally, shear stress-strain curves in $\{111\}<110>$ and $\{111\}<11 \overline{2}>$ directions were investigated using both the first principles calculations and the MD method.
\end{abstract}

This is the author's manuscript of the article published in final edited form as:

Guo, X., Park, H.-Y., Jung, Y.-G., \& Zhang, J. (2021). Atomistic modeling of anisotropic mechanical properties of lanthanum zirconate nanocystal. Materials Chemistry and Physics, 259, 124024. https://doi.org/10.1016/j.matchemphys.2020.124024 
Results show that Young's modulus of LZ crystal is highly anisotropic. The crystal has the highest Young's modulus in [111] direction, and $\{111\}<11 \overline{2}>$ direction is the favorable slip system during shear deformations.

Keywords: First principles; Molecular dynamics; Mechanical properties; Lanthanum Zirconate; Nanocrystal 


\section{Introduction}

Lanthanum zirconate $\left(\mathrm{La}_{2} \mathrm{Zr}_{2} \mathrm{O}_{7}\right.$, or $\left.\mathrm{LZ}\right)$ is a promising candidate material for thermal barrier coating (TBC), which has the potential applications in aerospace and power generation industries $[1,2]$. LZ has lower thermal conductivity and good phase stability than the present state-of-the-art $8 \mathrm{wt} \% \mathrm{Y}_{2} \mathrm{O}_{3}$-stabilized $\mathrm{ZrO}_{2}$ (8YSZ) [3].

It has been reported that LZ has a lower Young's modulus and lower fracture toughness than 8YSZ [3]. Also, the thermal cycling performance of the LZ single-layer coating is worse than that of 8YSZ, which might be due to LZ's low coefficient of thermal expansion (CTE) and poor mechanical properties $[4,5]$. We previously reported that the double-layer porous 8YSZ plus LZ coating had a better performance in thermal cycling experiments[6]. These coating layers were undertaken both tensile and shearing stresses during the thermal cycling process. To understand the failure mechanism of the coatings during the thermal cycling process, the analyses of the tensile and shearing stress-strain relationships are justified. Therefore, it is important to understand the mechanical response of LZ crystal in tensile and shear deformations. Because it is difficult to experimentally measure the mechanical properties of LZ nanocrystal, atomistic modeling becomes a valuable tool.

Several analytical methods have been employed to understand the theoretical elastic modulus of complicated ceramics like LZ [7-9]. These methods derive Young's modulus from the elastic constants, which can be computed by the first principles calculations based on the density functional theory (DFT) $[10,11]$. Akgenc et al studied the structure and mechanical properties of various $\mathrm{ABO}_{3}(\mathrm{~A}=\mathrm{Ba}, \mathrm{Sr}, \mathrm{Pb} ; \mathrm{B}=\mathrm{Ti}, \mathrm{Zr})$ perovskites and 6-15\% YSZ using

first principles calculations $[12,13]$. Liu et al. showed that Young's modulus of LZ crystal was anisotropic, using Ingel's anisotropic equation [7, 14]. Liu et al. reported that the highest Young's modulus was $252 \mathrm{GPa}$ in [111] direction, and the lowest was $214 \mathrm{GPa}$ in [100] direction. Feng et al. studied the mechanical properties of $\mathrm{Ln}_{2} \mathrm{Zr}_{2} \mathrm{O}_{7}$ (where $\mathrm{Ln}=\mathrm{La}, \mathrm{Pr}, \mathrm{Nd}$, $\mathrm{Sm}, \mathrm{Eu}$, and $\mathrm{Gd}$ ) using the local-density approximation of spin-polarized scheme (LSDA) $+\mathrm{U}$ calculations, in which $U$ was the empirical parameter called Hubbard energy [15, 16]. In Feng's work, the coexistence of spin and weak ferromagnetic states were considered. However, the LSDA+U correction was not used in $\mathrm{La}_{2} \mathrm{Zr}_{2} \mathrm{O}_{7}$ because $4 \mathrm{f}$ orbital of La was 
fully unoccupied. The Voigt-Reuss-Hill approximation was used in Feng's work to calculate the Young's modulus (208 GPa) and shear modulus (87 GPa) [9, 15].

Additionally, simulations of uniaxial tensile test and shear test can be conducted using the DFT and molecular dynamics (MD) calculations, and the simulated stress-strain curves can be used to derive the Young's and shear moduli. Umeno et al. performed the DFT tensile simulations in nanoscale Si films in (001) direction [17]. The uniaxial tensile strain was applied step wisely in the tensile direction. After the full relaxation at each strain stage, the tensile stress was computed using the DFT calculations. Zhang and Liu et al. conducted the nanoscale shear and tensile simulations to investigated the stress-strain relations of super hard $\mathrm{BC}_{3}$ using the DFT calculations [18]. A shear strain was applied step wisely by the change of the crystal's angle. In addition to DFT calculations, MD is another method to perform the tensile and shear deformation simulations. Dupont et al. investigated the shear stress-strain relations of a copper single crystal in different orientations at various temperatures using the MD method [19]. The shear stress was calculated from the stress tensors of the uniaxial compressed single crystal copper model. Dupont found that temperature only had minimal effect on the stress-strain relations in all directions.

Although the above-mentioned methods were used to conduct the nanoscale tensile and shear simulations, to our best knowledge, no systematical study of mechanical properties was performed on LZ crystal. In this paper, the nanoscale tensile and shear stress-strain relations of LZ single crystal were investigated using both the DFT and MD methods. The corresponding Young's moduli and shear moduli were calculated using two approaches: stress-strain curve analysis in large deformation and the Voigt-Reuss-Hill approximation in small deformation $[9,20]$. Toughness values were calculated based on the stress-strain curves as well. Finally, average Bader charge difference and charge density distribution were computed to explain the observed Young's moduli due to election interaction. 


\section{Calculation methods}

\subsection{Density functional theory calculations}

In this work, all of the DFT calculations were conducted using the Vienna Ab initio Simulation Program (VASP) [21, 22]. The exchange-correlation potential was specified using the PBE method of generalized gradient approximation (GGA) functional. The energy convergence tests have been carried out to determine the number of k-point and the cut-off energy for all the structures. The Brillouin-zone integration with the Monkhorst-Pack scheme in a k-point of $3 \times 3 \times 3$ is required to stabilize the structure and compute the mechanical properties. The total energy is converged at the cut-off energy of $500 \mathrm{eV}$ for all of the structures. A conjugate-gradient algorithm was used for ionic relaxation. The energy relaxation criterion for the electron was $10^{-6} \mathrm{eV}$ and the ionic relaxation convergence criterion was the total forces less than $0.01 \mathrm{eV} / \mathrm{A}$. The $\mathrm{LZ}$ crystal is a cubic pyrochlore structure, which belongs to the space group of $F d \overline{3} m$ [23]. There are four independent crystallographic atom sites, where $\mathrm{La}$ is at $16 \mathrm{~d}$ and $\left(\frac{1}{2}, \frac{1}{2}, \frac{1}{2}\right), \mathrm{Zr}$ is at $16 \mathrm{c}$ and $(0,0,0), \mathrm{O} 1$ is at $48 \mathrm{f}$ and $\left(\mathrm{x}, \frac{1}{8}, \frac{1}{8}\right)$ and $\mathrm{O} 2$ is at $8 \mathrm{~b}$ and $\left(\frac{3}{8}, \frac{3}{8}, \frac{3}{8}\right)$. The $\mathrm{x}$ value of $\mathrm{O} 1$ varies from 0.3125 to 0.375 . According to Tabira's experiments, 0.333 is chosen as the initial $\mathrm{x}$ value of $\mathrm{LZ}$ in this work [24]. The conventional LZ unit cell is shown in Fig. 1. The unit cell was reconstructed to align the (001), (011), and (111) surface perpendicular to the tensile axis, as shown in Fig. 2. The atom numbers of the (001), (011), and (111) model were 44, 44, and 66, respectively. The reconstructed LZ structures to different tensile orientations were relaxed before the tensile and shear deformation calculations. 


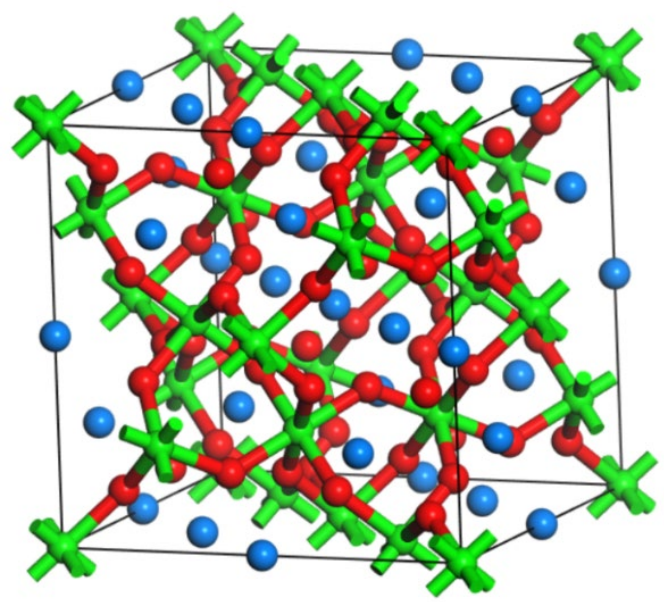

Fig. 1: Crystal structure of $\mathrm{La}_{2} \mathrm{Zr}_{2} \mathrm{O}_{7}$ unit cell. Balls are green, red, and blue for $\mathrm{Zr}$ atom, $\mathrm{O}$ atom, and La atom, respectively (same coloring schemes are used in all figures afterward).

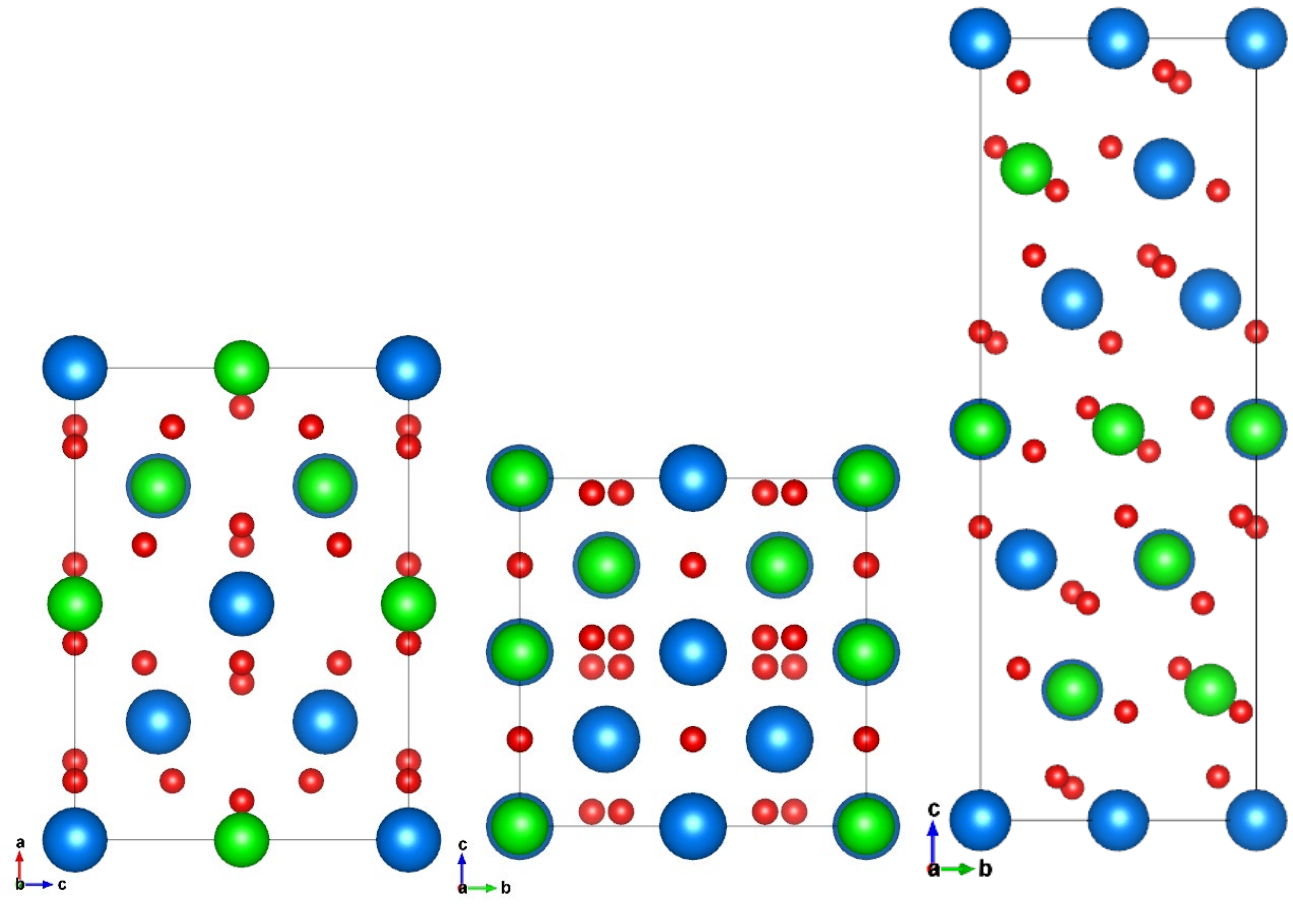

(a)

(b)

(c)

Fig. 2: Tensile deformation model in (a) [001] direction along a axis, (b) [011] direction along c axis, and (c) [111] direction along $\mathrm{c}$ axis.

The large deformation stress-strain analysis and the small deformation elastic constant analysis were used to calculate the anisotropic elastic moduli. In the stress-strain analysis, the anisotropic Young's modulus and shear modulus in a particular tensile or shear direction were obtained from the slope of the linearly fitted line in the elastic deformation of the stress-strain 
curves. In the small deformation elastic constant analysis, Voigt-Reuss-Hill approximation was used to calculate the elastic moduli of LZ based on the elastic constants of a single LZ unit cell. The elastic constants of the LZ unit cell were calculated using the DFT method by performing finite distortion of the unit cell [25]. The Voigt proposed approach expressed the stress in a single crystal in terms of the given strain. On the other hand, the Reuss proposed approach expressed the strain in terms of the given stress. The Voigt-Reuss-Hill approximation calculated the effective moduli of an aggregated polycrystalline using the combination of Voigt and Reuss's approximation [9, 26]. For the cubic LZ single crystal, only three elastic constants were independent $\left(\mathrm{C}_{11}, \mathrm{C}_{12}, \mathrm{C}_{44}\right)$. The effective bulk modulus $(K)$, shear modulus $(G)$, Young's modulus $(E)$, and Poisson's ratio $(v)$ of the cubic LZ crystal were calculated using the following equations:

$$
\begin{aligned}
& K=\frac{C_{11}+2 C_{22}}{3} \\
& G=\frac{G_{R}+G_{v}}{2} \\
& E=\frac{9 K G}{3 K+G} \\
& v=\frac{3 K-2 G}{2(3 K+G)}
\end{aligned}
$$

where $G_{V}$ and $G_{R}$ were the shear modulus expressed by the Voigt and Reuss approach, respectively $[9,26]$.

$$
\begin{aligned}
G_{V} & =\frac{\left(C_{11}-C_{22}\right)+3 C_{44}}{5} \\
\frac{5}{G_{R}} & =\frac{4}{C_{11}-C_{22}}+\frac{3}{C_{44}}
\end{aligned}
$$

The slip system (easy slip plane and direction) in the shearing process often occurs on the plane of high atomic density in closely packed directions [27]. For example, the primary slip system for face center cubic (fcc) crystal is $\{111\}<110>$, and the secondary slip direction is $\{111\}<11 \overline{2}>$. Because the $\{111\}$ is the closest packed plane in LZ crystal, the most possible slips are along $\{111\}<110>$ and $\{111\}<11 \overline{2}>$ directions. The conventional unit cell of LZ was used in the shear stress calculation, as shown in Fig. 1c. The shear directions were controlled by rotating the $\mathrm{LZ}$ unit cell to align the slip plane perpendicular to one of the coordinate axes. 
The shear strain was applied by changing the angle in the shear direction. The shear stress was calculated using the first principles calculations corresponding to each strain step.

Bader charge transfer analysis in the tensile and shear DFT models was conducted to describe the electron gain and loss between different atoms [28, 29]. The average charge differences between $\mathrm{Zr}$, La, and $\mathrm{O}$ atoms were calculated from the Bader charge results, which gave the insights of atom bonding character in different orientations.

\subsection{Molecular dynamics calculations}

All of the DFT calculations for tensile and shear were performed on the ground state of atoms, which was typically at a temperature of $0 \mathrm{~K}$. The tensile and shear simulations at higher temperatures were conducted using molecular dynamics (MD) calculations. Also, the MD calculations were performed at a larger length scale than the first principles calculations. All of the MD calculations in this study were conducted using LAMMPS (Large-scale Atomic/Molecular Massively Parallel Simulator) simulation package [30]. In this work, the Buckingham and Coulomb potentials were used to describe the short and long-range atom interactions, respectively, which were expressed as follows:

$$
U_{i j}\left(r_{i j}\right)=A_{i j} \exp \left(\frac{-r_{i j}}{\rho_{i j}}\right)-\frac{C_{i j}}{r_{i j}^{6}}+\frac{1}{4 \pi \epsilon_{0}} \cdot \frac{q_{i} q_{i} e^{2}}{r_{i j}}
$$

where $r_{i j}$ was the distance of the interactive ions, $q_{i}$ and $q_{j}$ were the charges of $\mathrm{La}^{3+}, \mathrm{Zr}^{4+}, \mathrm{O}^{2-}$ ions, $\rho$ was an ionic-pair dependent length parameter, and $\mathrm{A}$ and $\mathrm{C}$ were adjustable parameters. The parameters of the Buckingham potential used in this study are listed in Table 1, which were fitted based on experimental crystallographic data and Hartree-Fock calculated elastic constants by Crocombette et al. [31]. Both tensile and shear simulations were conducted at a temperature of $300 \mathrm{~K}$. The tensile simulation was carried out by applying uniaxial tensional loading along the $\mathrm{x}$-axis, and the shear simulation was performed by applying uniaxial compression loading. The simulation box was relaxed to allow its shape and size to change after any step of loading, to ensure that the stress was zero along with the loading transverse directions. A periodical boundary condition was used in all the MD simulations. The LZ unit cells shown in Fig. 2 were used to assembly the MD tensile and shear models. For tensile MD calculations, three tetragonal boxes of $12 \times 12 \times 24$ (individually in $\mathrm{x}, \mathrm{y}, \mathrm{z}$ 
directions) LZ unit cells in the [001], [110], and [111] directions were assembled as the tensile models in each direction. In the tensile model, the (001), (110), and (111) surfaces were perpendicular to the tensile axis $(\mathrm{z})$. The shear models were built with $16 \times 16 \times 16 \mathrm{LZ}$ unit cells in the [112] direction and $24 \times 36 \times 16 \mathrm{LZ}$ unit cell in the [110] direction. The compression axis was performed along the [110] and [112] directions. The conjugate gradient method was used to minimize the energy of the system in both tensile and shear simulations[32]. Then, the time integration was performed in isothermal-isobaric (NPT) ensembles, which was dedicated to generating the position and velocities parameters. Finally, the tensile models were elongated in the NPT ensembles, and the shear models were compressed in the canonical (NVT) ensembles. The time step of all the MD simulations performed is 1 picosecond. The total time length of the simulation process is $20 \mathrm{~ns}$ for the tensile test and $12 \mathrm{~ns}$ for the shear test.

Table 1: Buckingham potential parameter for LZ crystal [31].

\begin{tabular}{|c|c|c|c|c|}
\hline Interactions & $\mathrm{A}(\mathrm{eV})$ & $\mathrm{P}(\AA)$ & $\mathrm{C}\left(\mathrm{eV} \cdot \AA^{6}\right)$ & Charges \\
\hline $\mathrm{O}-\mathrm{O}$ & 22764.00 & 0.1490 & 27.89 & -2.0 \\
\hline La-O & 1367.41 & 0.3591 & 0.00 & -3.0 \\
\hline Zr-O & 1478.69 & 0.3554 & 0.00 & +4.0 \\
\hline
\end{tabular}

\section{Results and discussion}

\subsection{Large deformation stress-strain analysis}

The ions positions of the DFT models were relaxed using the conjugate-gradient algorithm before and during the deformation calculations. The model was the uniaxial loaded in the zdirection, so the tensile stress was acquired directly from the stress tensor in the z-direction. Fig. 3 shows the tensile stress-strain curves calculated using the first principles calculations. The linear elastic stage on these curves only occurred in the first few steps, then the stress dropped slowly after it reached the ultimate tensile strength. The LZ tensile model in the [001] direction had the largest ultimate tensile strength, and the LZ tensile models in the [110] and [111] directions had a similar ultimate strength. Young's modulus was calculated from the slope of the linear section in the curve using Hooke's law, and toughness was calculated from the integration of the area under the stress-strain curve. The calculated Young's modulus, 
ultimate tensile strength, and toughness are summarized in Table 2. The single crystal LZ had the maximum tensile Young's modulus in [111] direction and minimum Young's modulus in the [001] plane. This trend was consistent with Liu 's results [14]. However, the LZ tensile model in the [001] direction possessed the maximum ultimate strength and toughness.

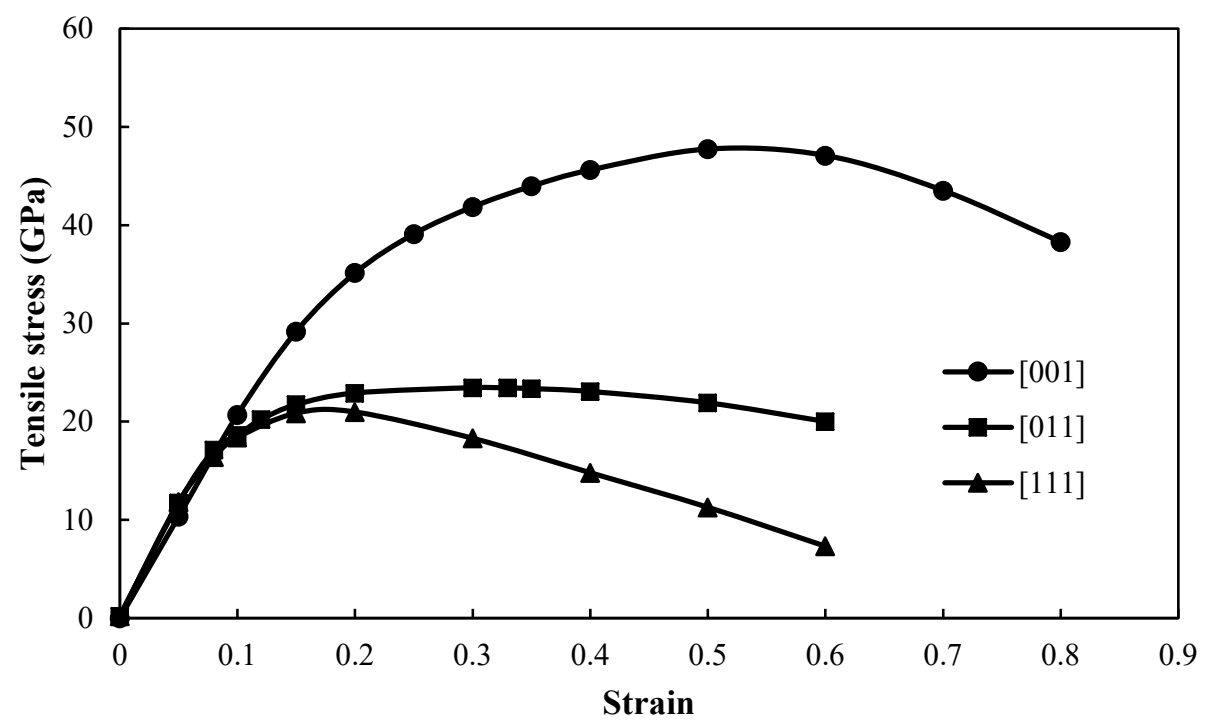

Fig. 3: DFT calculated tensile stress-strain curves

Fig. 4 shows the DFT calculated shear stress-strain curves in two slip systems $\{111\}<11 \overline{2}>$ and $\{111\}<110>$. The two curves are very similar. As summarized in Table 2, the shear modulus of $\{111\}<110>$ is slightly larger than that of $\{111\}<11 \overline{2}>$, and so as the ultimate shear strength and the toughness, although the differences are very small. When the two slip systems of LZ crystal are under the same stress level, the corresponding strain in $\{111\}<11 \overline{2}>$ direction will be larger than that in $\{111\}<110>$ direction. Based on these DFT calculated results, in single crystal LZ material, the $\{111\}<11 \overline{2}>$ direction system is more likely to slip in the shear simulation. 


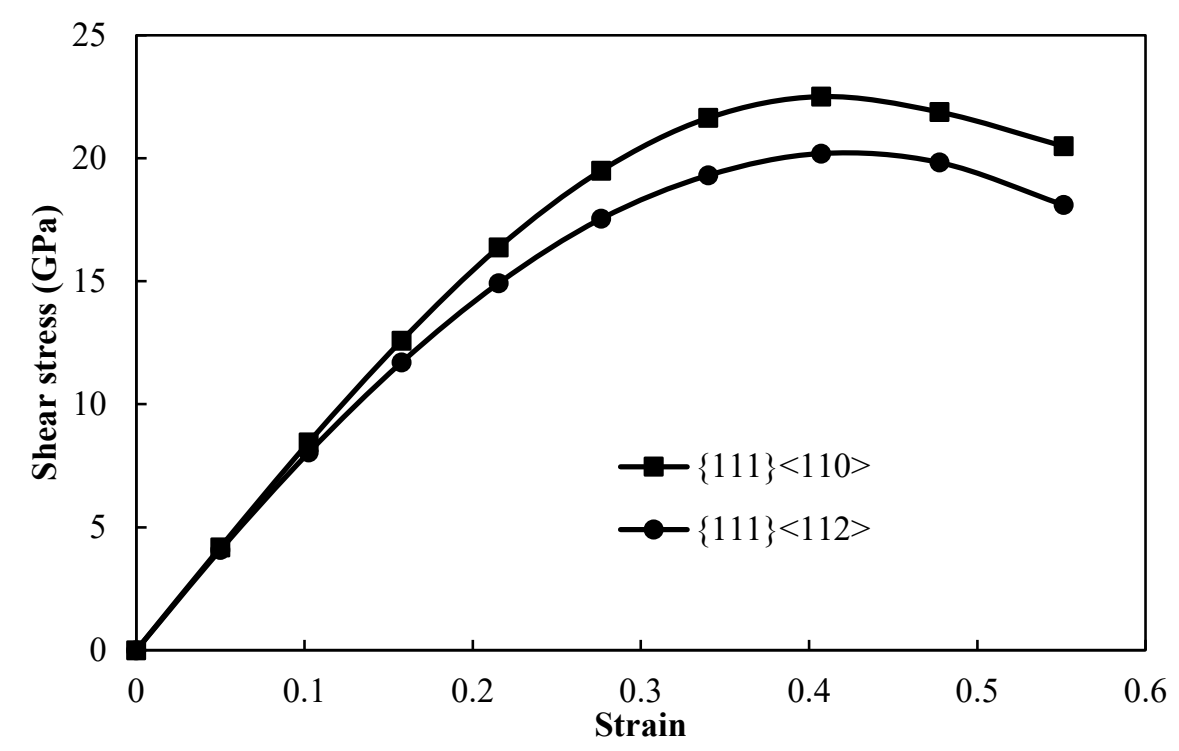

Fig. 4: DFT calculated shear stress-strain curves

Table 2: The calculated Young's modulus, ultimate strength, and toughness

\begin{tabular}{|c|c|c|c|}
\hline calculation model & $\begin{array}{c}\text { Young's modulus } \\
(\mathrm{GPa})\end{array}$ & $\begin{array}{c}\text { Ultimate strength } \\
(\mathrm{GPa})\end{array}$ & Toughness $\left(\mathrm{MJ} / \mathrm{m}^{3}\right)$ \\
\hline DFT Tensile [001] & 188.139 & 47.747 & 21.565 \\
\hline DFT Tensile [110] & 189.556 & 23.453 & 12.219 \\
\hline DFT Tensile [111] & 208.462 & 20.980 & 8.934 \\
\hline MD Tensile [001] & 255.913 & 30.250 & 3.018 \\
\hline MD Tensile [110] & 224.109 & 18.867 & 2.192 \\
\hline MD Tensile [111] & 207.219 & 18.914 & 1.839 \\
\hline DFT Shear $\{111\}<110>$ & 76.037 & 22.507 & 8.862 \\
\hline DFT Shear $\{111\}<11 \overline{2}>$ & 73.381 & 20.192 & - \\
\hline MD Shear $\{111\}<110>$ & - & 29.008 & - \\
\hline MD Shear $\{111\}<11 \overline{2}>$ & - & 20.774 & \\
\hline
\end{tabular}

\subsection{Small deformation Young's moduli calculation}

The elastic constants $\mathrm{C}_{11}, \mathrm{C}_{12}$, and $\mathrm{C}_{44}$ of the $\mathrm{LZ}$ single crystal were calculated using the DFT method in small deformation conditions, which are 256.001 GPa, 116.425 GPa, and 
86.708 $\mathrm{GPa}$, respectively, which are comparable to the previous studies $\left(\mathrm{C}_{11}=291.6\right.$, $\mathrm{C}_{12}=126.3$, and $\left.\mathrm{C}_{44}=99.6 \mathrm{GPa}\right)[33]$.

The effective elastic moduli of LZ were calculated using equations (1)-(6), as summarized in Table 3. Some experiments results from the literature are also listed in

Table 3. As a comparison, Zhang et al. measured Young's modulus of 7\% porous LZ coating using nano-indentation technology[34]. Shimamura et al. studied the moduli of LZ materials using the ultrasound pulse-echo measurement [35]. Guo et al. studied the mechanical properties of LZ based coatings [36-40]. Compared with the experiment data, the effective moduli of the LZ were in reasonably good agreement.

Table 3: Effective moduli of LZ and the experimental results

\begin{tabular}{|c|c|c|}
\hline & Effective moduli in this study & Experimental results \\
\hline Young's modulus $(\mathrm{GPa})$ & 205.12 & $156 \pm 10(\mathrm{Zhang}[34])$ \\
$280($ Shimamura[35])
\end{tabular}

The anisotropic characteristics can be determined by Zener anisotropic ratio, which is defined as $Z=2 C_{44} /\left(C_{11}-C_{22}\right)$ [7]. If $Z=1$, the material is isotropic. The calculated $Z$ value for LZ was 1.24, indicating that the elastic moduli of LZ were anisotropic, and the maximum Young's modulus was in $<111>$ direction. Anisotropic Young's modulus of cubic LZ crystal was expressed using the DFT calculated elastic constants, which was given by the following equations [7].

$$
\begin{aligned}
& \frac{1}{E}=\frac{l^{4}+m^{4}+n^{4}}{E_{0}}+\frac{2\left(m^{2} n^{2}+n^{2} l^{2}+l^{2} m^{2}\right)}{F_{0}} \\
& \frac{1}{E_{0}}=\frac{C_{11}+C_{22}}{\left(C_{11}-C_{22}\right)\left(C_{11}+2 C_{22}\right)} \\
& \frac{2}{F_{0}}=\frac{-2 C_{12}}{\left(C_{11}-C_{22}\right)\left(C_{11}+2 C_{22}\right)}+\frac{1}{C_{44}}
\end{aligned}
$$


where $l, m, n$ were the directional cosines. The (110) plane was chosen in this work for the anisotropic calculation because it included all three principal directions in a cubic crystal $<111>,<110>$, and $<001>$. The directional cosines for (110) plane were calculated by:

$l=\cos \theta \quad m=\frac{\sin \theta}{\sqrt{2}} \quad n=\frac{\sin \theta}{\sqrt{2}}$

where $\theta$ was the angle in the (110) plane, which was measured from the [100] to [110] direction.

The anisotropic Young's modulus results are plotted in Fig. 5, including the solid curve calculated from the elastic constants of LZ single crystal in small deformation status, and the scattered points calculated from the DFT and the MD (the MD results will be discussed in section 3.4) stress-strain curves in the large deformation simulations. As shown in Fig. 5, most of anisotropic Young's modulus results are very close to each other, suggesting that this modeling study has good consistency and accuracy.

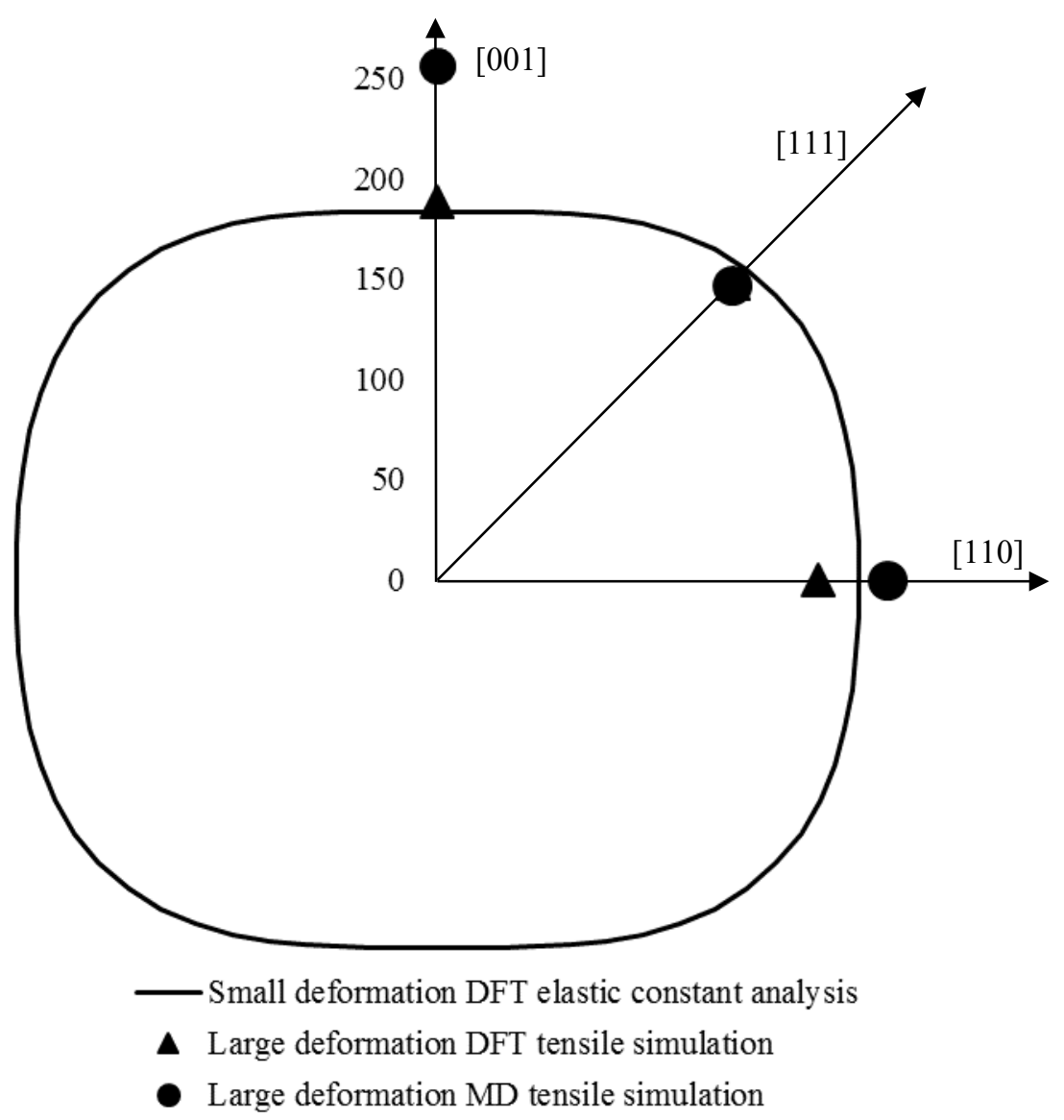


Fig. 5 Summary of anisotropic Young's moduli using DFT and MD.

\subsection{Bader charge analysis and charge density distribution}

Bader charge transfer was calculated considering the charge transfer of each atom in the tensile and shear deformation systems. The unit of Bader charge is an electron (e), which means that the number of electrons that the atom gains or losses. The Bader charge differences between cation and anion resulting from each tensile and shear calculations were summarized in Table 4. The average Bader charge difference between cation and anion reveals the electron interaction between the two atoms, which is related to the binding character of the atoms. A high average Bader charge difference indicates a strong bond. The charge differences between $\mathrm{Zr}$ and $\mathrm{O}$, and between $\mathrm{La}$ and $\mathrm{O}$ atoms in tensile [111] calculation are the largest among the three tensile cases, suggesting that the bonding strength in the tensile model at the [111] direction is the strongest. The strong bond between the cation and anion in the [111] tensile model corresponds to the high Young's modulus results in DFT calculations. In the shear models, the average Bader charge difference in the LZ shearing model in $\{111\}<110>$ direction is slightly larger than that in $\{111\}<11 \overline{2}>$ direction, suggesting the atom bond in $\{111\}<110>$ shear model is stronger than that in $\{111\}<11 \overline{2}>$ direction. The stronger bond of the shear model in the $\{111\}<110>$ direction results in a higher Young's modulus than that of $\{111\}<11 \overline{2}>$ direction.

Table 4: Calculated average Bader charge difference between $\mathrm{O}, \mathrm{Zr}$, and La atoms

\begin{tabular}{|c|c|c|}
\hline & $\begin{array}{c}\text { Average Bader Charge difference } \\
\text { between } \mathrm{O} \text { and } \mathrm{Zr}\end{array}$ & $\begin{array}{c}\text { Average Bader Charge difference } \\
\text { between O and La }\end{array}$ \\
\hline Tensile in [001] & 4.592 & 3.469 \\
\hline Tensile in [110] & 4.804 & 3.603 \\
\hline Tensile in [111] & 5.106 & 3.696 \\
\hline Shear in $\{111\}<110>$ & 4.965 & 3.568 \\
\hline Shear in $\{111\}<11 \overline{2}>$ & 4.893 & 3.535 \\
\hline
\end{tabular}


The contours of charge density distribution in each LZ tensile model with the strain of 0.5 are plotted in Fig. 6. Charge density distribution reveals the election loss and gains property. The red color indicates election gain and the blue color indicates the election loss. As shown in Fig. 6, the $\mathrm{Zr}$ atoms lost most of their valence electrons to the $\mathrm{O}$ atoms. The density distributions near the $\mathrm{O}$ atoms were similar in the [001] tensile model and [110] tensile model. However, the [111] tensile model had the highest charge density near the $\mathrm{O}$ atom among all the tensile models, suggesting the electron interaction in the [111] tensile model is the strongest. This strong election interaction leads to a high Young's modulus in the [111] direction.

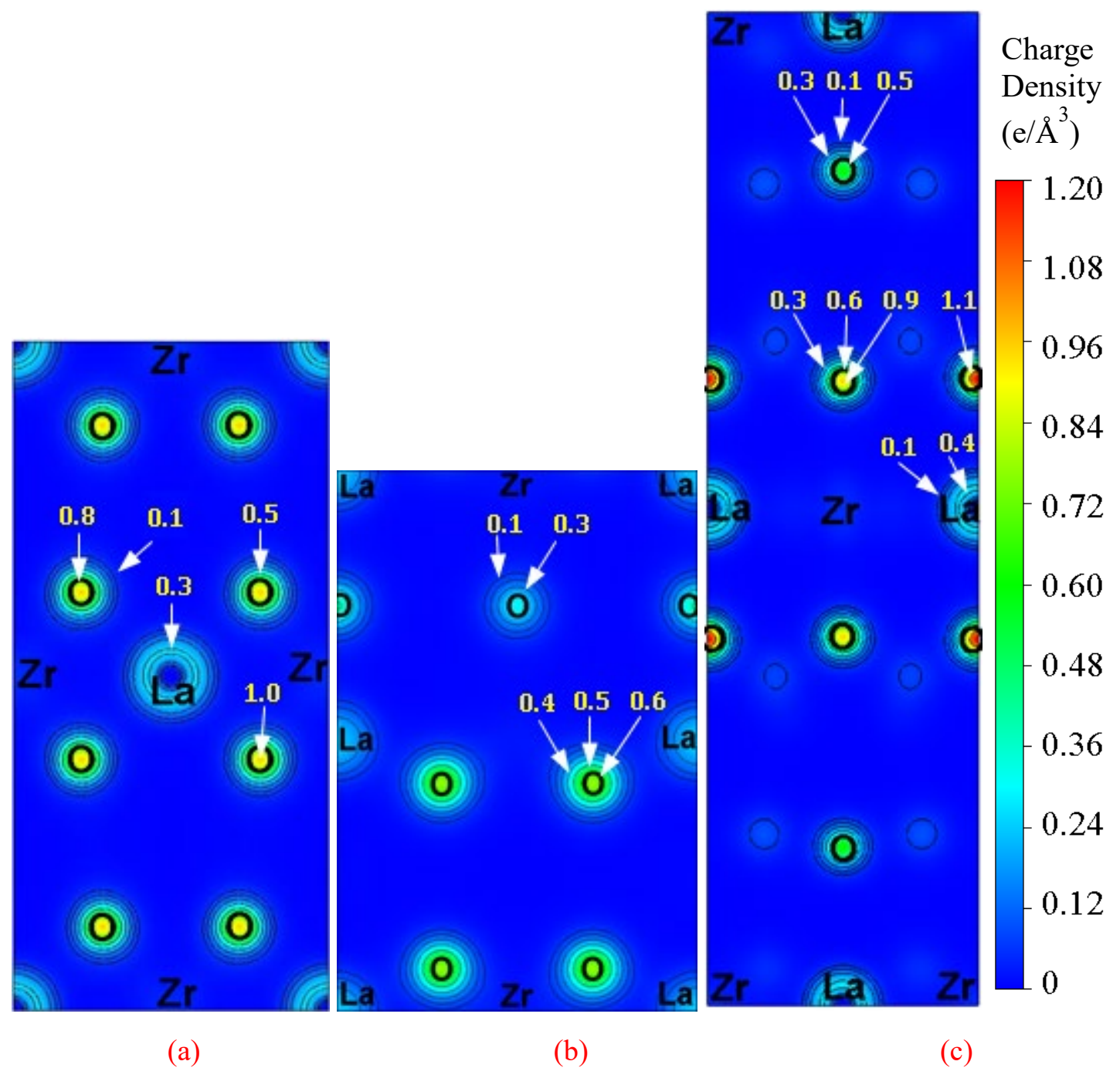

Fig. 6: Charge density distribution $\left(\mathrm{e} / \AA^{3}\right)$ of tensile calculation at the strain of 0.5 in (a) [001] direction, (b) [110] direction and (c) [111] direction. 


\subsection{MD tensile and shear simulations}

Fig. 7 shows the models of the first and last steps of the MD tensile calculations in the [001] direction. The model image of the initial step demonstrates the perfect LZ single crystal without any tensile strain. The model image of the 0.16 strain step shows that there are some vastly deformed areas in the model, which reach its ultimate strength and it's about to break apart.

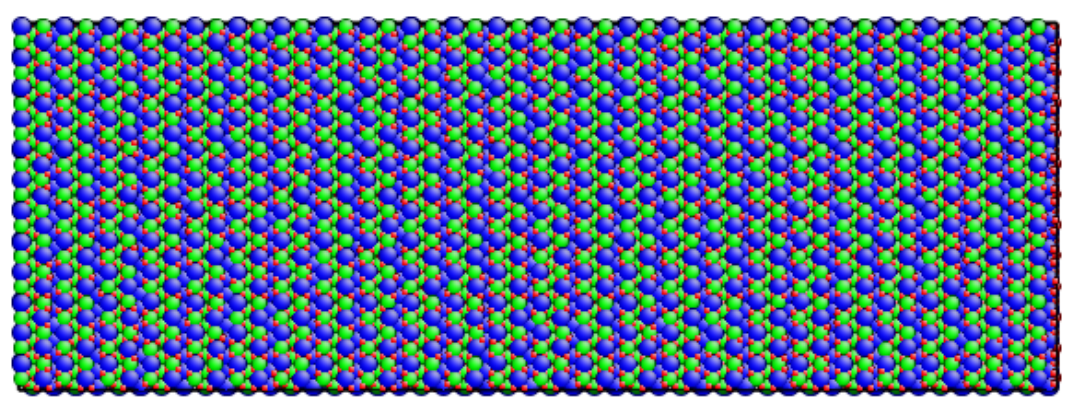

(a)

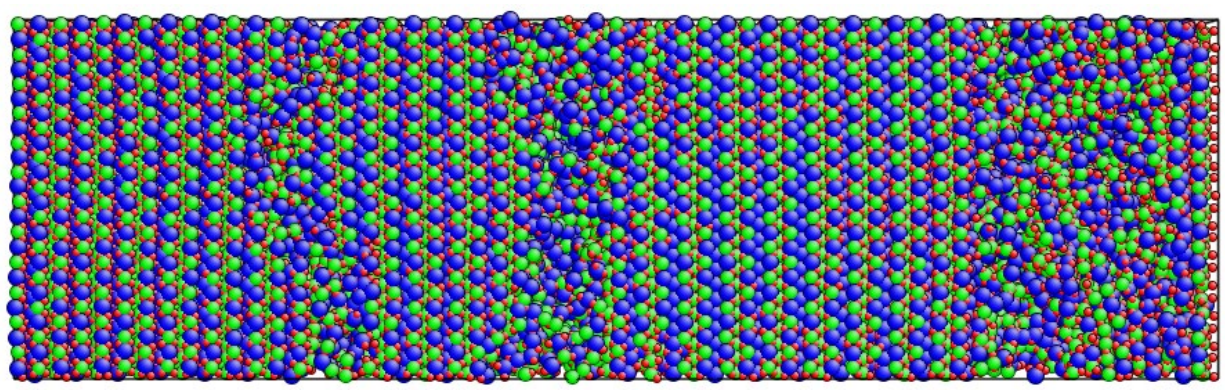

(b)

Fig. 7: MD calculated tensile model in [001] direction: (a) the initial step (no strain);and (b) the step with 0.16 strain. Green, red, and blue balls indicate $\mathrm{Zr}$ atom, $\mathrm{O}$ atom, and La atom, respectively.

Fig.8 shows the MD calculated tensile stress-strain curves in [111], [001], [011] directions. All of the stress-strain curves in these three tensile simulations exhibited an elastic stage and the stress dropped after the maximum tensile stresses were reached. The slops of these stressstrain curves in the elastic stage were very similar to the beginning stage and varied as the increase of the stress. The Young's moduli of LZ in the [111], [001], [011] directions were calculated from the slope of linear parts, which were similar, as summarized in Table 2. However, the stress-strain curve in the [111] direction showed a longer linear elastic stage and higher ultimate strength than the stress-strain curves in the other two directions, indicating 
that LZ had a larger toughness in the [111] direction than those in the [110] and [001] directions. The MD calculated ultimate tensile strength shared the same trend with the DFT results.

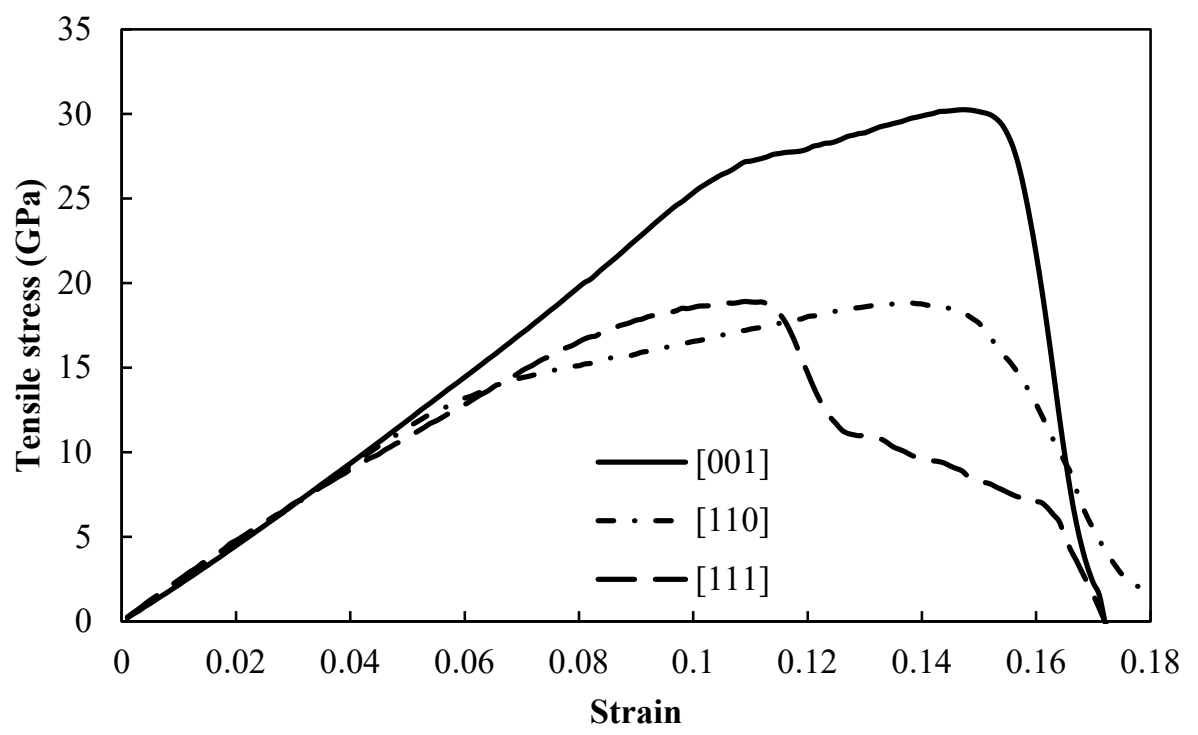

Fig. 8: The MD calculated tensile stress-strain curves of LZ in [001],[011], and [111] directions.

The shearing stress was derived from the virial theory by averaging the virial stresses over the whole system, which means only the potential energies should be considered [41]. The shear stress was calculated by the following equation:

$$
\tau=-0.5\left[\sigma_{x x}-0.5\left(\sigma_{y y}+\sigma_{z z}\right)\right]
$$

where $x$ was the uniaxial compression axis [19]. The relation between the resolved compression and shear slip system was also considered. The orientation of the slip plane in the LZ normal compression model was very close to the initial compression axis, so the compressive shear strain was used to specify the strain in the slip plane as a simplification [19].

Fig. 9 shows the MD calculated shear stress-strain curves in [110] and [112] directions. The shear stress-strain curves have a similar form as the tensile stress-strain curve, which shows a linear elastic stage. The ultimate shear strength of [110] is larger than that of [112]. Similar to the DFT calculated shear stress-strain curves, the MD shear results also indicate that LZ single crystal in [112] direction is more likely to slip than in [110] direction. 


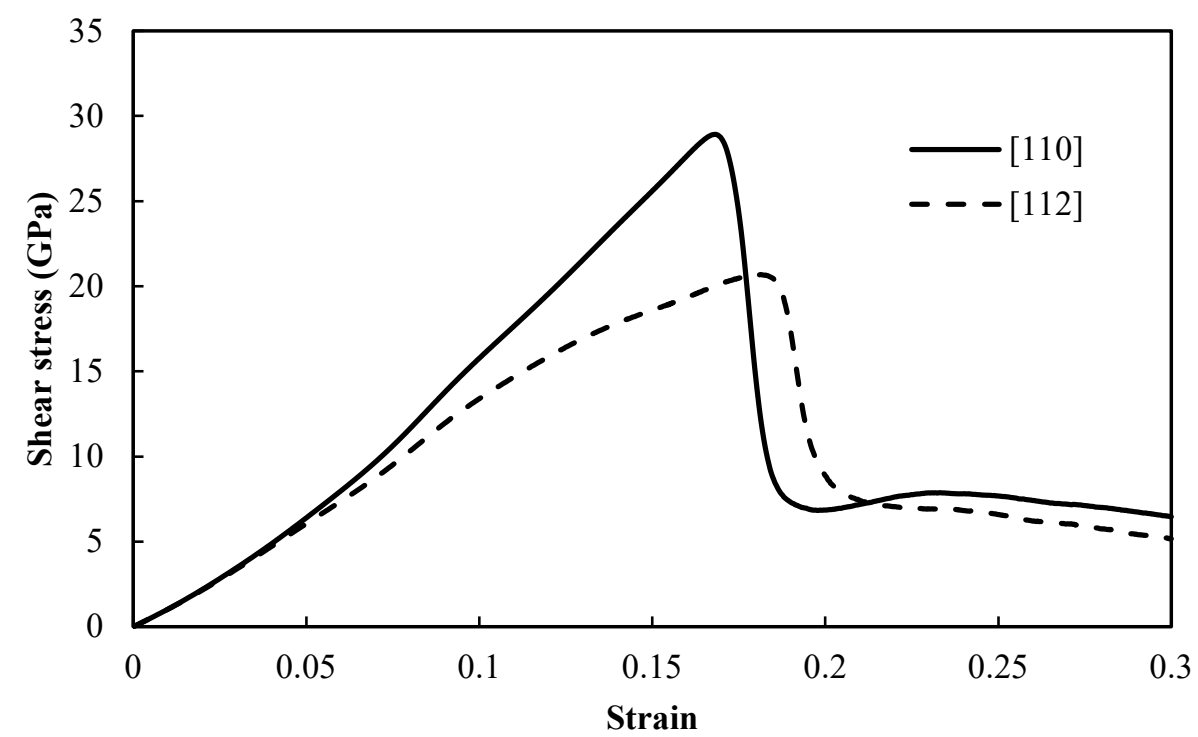

Fig. 9: MD calculated shear stress-strain curves of LZ in [110] and [112] directions

\section{Conclusions}

In this work, the nanoscale tensile and shear simulations for LZ single crystal material were performed using both the DFT and MD calculations. The corresponding mechanical properties were calculated using two approaches: stress-strain curve analysis in large deformation and Voigt-Reuss-Hill approximation in small deformation. The DFT calculated results of both methods were very close, suggesting the consistency of these two methods. Compared with the experimental results from the literature, the effective elastic moduli calculated from Voigt-Reuss-Hill approximation had good accuracy. The conclusions can be summarized as follows:

(1) The elastic moduli of LZ single crystal were anisotropic. The DFT results showed that the largest Young's modulus and the lowest ultimate tensile strength and toughness occurred in [111] direction. The average Bader charge difference analysis indicated that the bonds between $\mathrm{Zr}$ and $\mathrm{O}$, and between $\mathrm{La}$ and $\mathrm{O}$ were the strongest in LZ tensile model in [111] direction. The charge density distribution analysis exhibited the strong electron interaction in the tensile [111] model, which led to a high Young's modulus. 
(2) The DFT calculated shear stress-strain curves in $\{111\}<110>$ and $\{111\}<11 \overline{2}>$ slip systems were very similar, so as the average Bader charge difference between the cations and anions in these two models. However, the most likely slip system in $\{111\}$ plane was $\{111\}<11 \overline{2}>$, because more strain was generated in $\{111\}<11 \overline{2}>$ shearing model than that in $\{111\}<110>$ shearing model under the same stress level.

\section{Acknowledgments}

This work is partially supported by "Human Resources Program in Energy Technology (No. 20194030202450)" and "Power Generation \& Electricity Delivery grant (No. 20193310100030)" of the Korea Institute of Energy Technology Evaluation and Planning (KETEP). Xingye Guo would like to thank the financial support from the National Nature Science Foundation of China (grant number 51901006). 


\section{References}

[1] R. Vaßen, M. O. Jarligo, T. Steinke, D. E. Mack, and D. Stöver, "Overview on advanced thermal barrier coatings," Surface and Coatings Technology, vol. 205, no. 4, pp. 938-942, 11/15/2010.

[2] D. R. Clarke, M. Oechsner, and N. P. Padture, "Thermal-barrier coatings for more efficient gas-turbine engines," MRS Bulletin, vol. 37, no. 10, pp. 891-898, 2012.

[3] R. Vassen, X. Cao, F. Tietz, D. Basu, and D. Stöver, "Zirconates as New Materials for Thermal Barrier Coatings," Journal of the American Ceramic Society, vol. 83, no. 8, pp. 2023-2028, 2000.

[4] R. Vaßen, F. Traeger, and D. Stöver, "New Thermal Barrier Coatings Based on Pyrochlore/YSZ Double-Layer Systems," International Journal of Applied Ceramic Technology, vol. 1, no. 4, pp. 351-361, 2004.

[5] X. Guo, Z. Lu, Y.-G. Jung, L. Li, J. Knapp, and J. Zhang, "Thermal Properties, Thermal Shock, and Thermal Cycling Behavior of Lanthanum Zirconate-Based Thermal Barrier Coatings," Metallurgical and Materials Transactions E, journal article vol. 3, no. 2, pp. 64-70, 2016.

[6] X. Guo et al., "Thermal properties of La2Zr2O7 double-layer thermal barrier coatings," Advances in Applied Ceramics, vol. 118, no. 3, pp. 91-97, 2019/04/03 2019.

[7] R. P. Ingel and D. L. Iii, "Elastic Anisotropy in Zirconia Single Crystals," Journal of the American Ceramic Society, vol. 71, no. 4, pp. 265-271, 1988.

[8] O. L. Anderson, "A simplified method for calculating the Debye temperature from elastic constants," Journal of Physics and Chemistry of Solids, vol. 24, no. 7, pp. 909-917, 1963.

[9] R. Hill, "The elastic behaviour of a crystalline aggregate," Proceedings of the Physical Society. Section A, vol. 65, no. 5, p. 349, 1952.

[10] P. Hohenberg and W. Kohn, "Inhomogeneous electron gas," Physical review, vol. 136, no. 3B, p. B864, 1964.

[11] W. Kohn and L. J. Sham, "Self-Consistent Equations Including Exchange and Correlation Effects," Physical Review, vol. 140, no. 4A, pp. A1133-A1138, 11/15/ 1965.

[12] B. Akgenc, A. Kinaci, C. Tasseven, and T. Cagin, "First - principles calculations on stability and mechanical properties of various ABO3 and their alloys," Materials Chemistry and Physics, vol. 205, pp. 315-324, 2018/02/01/ 2018.

[13] B. Akgenç and T. Çağin, "Density functional theory of cubic zirconia and 6-15 mol\% doped yttria-stabilized zirconia: structural and mechanical properties," Turkish Journal of Physics, vol. 42, no. 2, 2018.

[14] B. Liu, J. Y. Wang, Y. C. Zhou, T. Liao, and F. Z. Li, "Theoretical elastic stiffness, structure stability and thermal conductivity of La2Zr2O7 pyrochlore," Acta Materialia, vol. 55, no. 9, pp. 2949-2957, 2007.

[15] J. Feng et al., "Electronic structure, mechanical properties and thermal conductivity of Ln2Zr2O7 (Ln=La, Pr, Nd, Sm, Eu and Gd) pyrochlore," Acta Materialia, vol. 59, no. 4, pp. 1742-1760, 2// 2011.

[16] V. I. Anisimov, J. Zaanen, and O. K. Andersen, "Band theory and Mott insulators: Hubbard U instead of Stoner I," Physical Review B, vol. 44, no. 3, p. 943, 1991. 
[17] Y. Umeno, A. Kushima, T. Kitamura, P. Gumbsch, and J. Li, "Ab initio study of the surface properties and ideal strength of (100) silicon thin films," Physical Review B, vol. 72, no. 16, p. 165431, 10/31/2005.

[18] M. Zhang et al., "Superhard BC 3 in cubic diamond structure," Physical review letters, vol. 114, no. 1, p. 015502, 2015.

[19] V. Dupont and T. C. Germann, "Strain rate and orientation dependencies of the strength of single crystalline copper under compression," Physical Review B, vol. 86, no. 13, p. 134111, 2012.

[20] L. Wu and J. Zhang, "Ab initio study of anisotropic mechanical properties of LiCoO2 during lithium intercalation and deintercalation process," Journal of Applied Physics, vol. 118, no. 22, p. 225101, 2015.

[21] G. Kresse and J. Furthmüller, "Efficient iterative schemes for ab initio total-energy calculations using a plane-wave basis set," Physical Review B, vol. 54, no. 16, pp. 11169-11186, 10/15/ 1996.

[22] G. Kresse and J. Furthmüller, "Efficiency of ab-initio total energy calculations for metals and semiconductors using a plane-wave basis set," Computational Materials Science, vol. 6, no. 1, pp. 15-50, 7// 1996.

[23] T. Moriga, A. Yoshiasa, F. Kanamaru, K. Koto, M. Yoshimura, and S. Sōmiya, "Crystal structure analyses of the pyrochlore and fluorite-type $\mathrm{Zr} 2 \mathrm{Gd} 2 \mathrm{O} 7$ and antiphase domain structure," Solid State Ionics, vol. 31, no. 4, pp. 319-328, 1// 1989.

[24] Y. Tabira, R. L. Withers, L. Minervini, and R. W. Grimes, "Systematic Structural Change in Selected Rare Earth Oxide Pyrochlores as Determined by Wide-Angle CBED and a Comparison with the Results of Atomistic Computer Simulation," Journal of Solid State Chemistry, vol. 153, no. 1, pp. 16-25, 8// 2000.

[25] G. Kresse, M. Marsman, and J. Furthmüller, "VASP the guide," http://cms.mpi.univie.ac.at/vasp/vasp/, 9.9. 2013.

[26] J. Den Toonder, J. Van Dommelen, and F. Baaijens, "The relation between single crystal elasticity and the effective elastic behaviour of polycrystalline materials: theory, measurement and computation," Modelling and Simulation in Materials Science and Engineering, vol. 7, no. 6, p. 909, 1999.

[27] N. K. Arakere and G. Swanson, "Effect of crystal orientation on fatigue failure of single crystal nickel base turbine blade superalloys," in ASME Turbo Expo 2000: Power for Land, Sea, and Air, 2000, pp. V004T01A004-V004T01A004: American Society of Mechanical Engineers.

[28] W. Tang, E. Sanville, and G. Henkelman, "A grid-based Bader analysis algorithm without lattice bias," Journal of Physics: Condensed Matter, vol. 21, no. 8, p. 084204, 2009.

[29] G. Henkelman, A. Arnaldsson, and H. Jónsson, "A fast and robust algorithm for Bader decomposition of charge density," Computational Materials Science, vol. 36, no. 3, pp. 354-360, 2006.

[30] S. Plimpton, "Fast Parallel Algorithms for Short-Range Molecular Dynamics," Journal of Computational Physics, vol. 117, no. 1, pp. 1-19, 3/1/ 1995.

[31] J.-P. Crocombette and A. Chartier, "Molecular dynamics studies of radiation induced phase transitions in La $2 \mathrm{Zr} 2 \mathrm{O} 7$ pyrochlore," Nuclear Instruments and Methods in Physics Research Section B: Beam Interactions with Materials and Atoms, vol. 255, no. 1, pp. 158-165, 2007. 
[32] M. R. Hestenes and E. Stiefel, Methods of conjugate gradients for solving linear systems. NBS, 1952.

[33] J. Yang, M. Shahid, M. Zhao, J. Feng, C. Wan, and W. Pan, "Physical properties of La2B2O7(BZr, Sn, Hf and Ge) pyrochlore: First-principles calculations," Journal of Alloys and Compounds, vol. 663, pp. 834-841, 2016/04/05/ 2016.

[34] J. Zhang, X. Guo, Y.-G. Jung, L. Li, and J. Knapp, "Microstructural non-uniformity and mechanical property of air plasma-sprayed dense lanthanum zirconate thermal barrier coating," Materials Today: Proceedings, vol. 1, no. 1, pp. 11-16, 2014.

[35] K. Shimamura, T. Arima, K. Idemitsu, and Y. Inagaki, "Thermophysical Properties of Rare-Earth-Stabilized Zirconia and Zirconate Pyrochlores as Surrogates for Actinide-Doped Zirconia," (in English), International Journal of Thermophysics, vol. 28, no. 3, pp. 1074-1084, 2007/06/01 2007.

[36] J. Zhang and Y.-G. Jung, Advanced Ceramic and Metallic Coating and Thin Film Materials for Energy and Environmental Applications. Springer International Publishing, 2018.

[37] J. Zhang et al., "Mechanical properties of lanthanum zirconate-based composite thermal barrier coatings," Advances in Applied Ceramics, vol. 118, no. 5, pp. 257263, 2019/07/04 2019.

[38] X. Guo, H.-M. Park, L. Li, J. Knapp, Y.-G. Jung, and J. Zhang, "Mechanical Properties of Layered La2Zr2O7 Thermal Barrier Coatings," Journal of Thermal Spray Technology, pp. https://doi.org/10.1007/s11666-018-0703-5 2018.

[39] J. Zhang, X. Guo, Y.-G. Jung, L. Li, and J. Knapp, "Lanthanum Zirconate Based Thermal Barrier Coatings: A Review," Surface and Coatings Technology, 2016.

[40] D. Song et al., "Microstructure design for blended feedstock and its thermal durability in lanthanum zirconate based thermal barrier coatings," Surface and Coatings Technology, vol. 308, pp. 40-49, 2016.

[41] E. T. Lilleodden, J. A. Zimmerman, S. M. Foiles, and W. D. Nix, "Atomistic simulations of elastic deformation and dislocation nucleation during nanoindentation," Journal of the Mechanics and Physics of Solids, vol. 51, no. 5, pp. 901-920, 5// 2003. 